\title{
Directed-loop Monte Carlo simulations of vertex models
}

\author{
Olav F. Syljuåsen ${ }^{1}$ and M. B. Zvonarev ${ }^{2}$ \\ ${ }^{1}$ NORDITA, Blegdamsvej 17, Copenhagen Ø, DK-2100 Denmarl $*$ \\ ${ }^{2}$ Ørsted Laboratory, Niels Bohr Institute for APG, \\ Universitetsparken 5, Copenhagen Ø, DK 2100, Denmart
}

(Dated: November 5, 2018)

\begin{abstract}
We show how the directed-loop Monte Carlo algorithm can be applied to study vertex models. The algorithm is employed to calculate the arrow polarization in the six-vertex model with the domain wall boundary conditions (DWBC). The model exhibits spatially separated ordered and "disordered" regions. We show how the boundary between these regions depends on parameters of the model. We give some predictions on the behavior of the polarization in the thermodynamic limit and discuss the relation to the Arctic Circle theorem.
\end{abstract}

PACS numbers: 05.50.+q, 05.10.Ln, 02.30.Ik

\section{INTRODUCTION}

Vertex models have a long and distinguished history in physics. Their fame is intimately connected to the concept of integrability, and the exact solutions of the six-vertex [1] and the eight-vertex [2] models with periodic boundary conditions (PBC) are indeed milestones in physics. Despite being exactly solvable, there are questions about these models that cannot easily be answered. An example is the influence of boundary conditions on correlation functions. While boundary conditions are not normally important in the thermodynamic limit, they have a profound influence on the vertex models. Exact studies, made for the six-vertex model with the domain wall boundary conditions (DWBC) [3] show this in particular. These studies were restricted to certain points in the phase diagram, and involve rather sophisticated mathematical methods. It is thus appropriate to complement them with Monte Carlo simulations.

The purpose of this article is to demonstrate that the directed-loop Monte Carlo algorithm developed for quantum spin systems [4] can be used as an effective tool to study vertex models. The discussion of the algorithm will be kept general, but when demonstrating its use we will focus on the six-vertex model with the DWBC, a model which is difficult to simulate using other known Monte Carlo algorithms.

\section{MONTE CARLO ALGORITHM}

In a vertex model, each vertex have edges with an Ising-like variable, an arrow, that points either away from or into the vertex. The arrangement of arrows around the vertex determines the vertex weight. Two vertices are joined by their common edge, sharing the arrow on the edge. In general there are no restrictions on which

*Electronic address: sylju@nordita.dk

${ }^{\dagger}$ Electronic address: zvonarev@fys.ku.dk

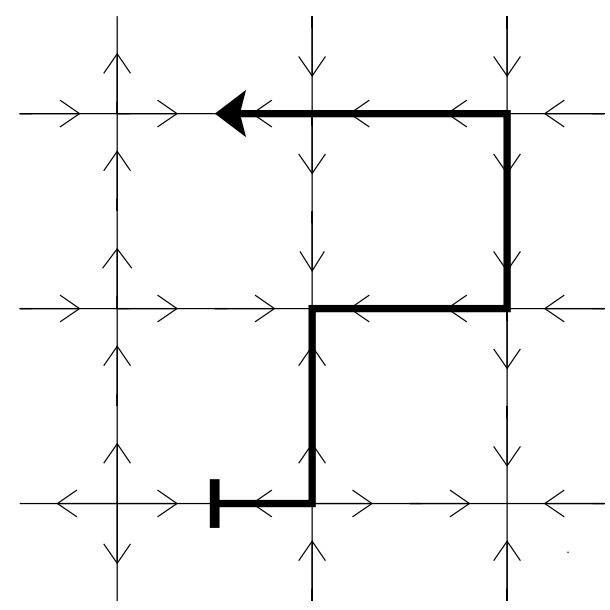

FIG. 1: Illustration of the directed-loop algorithm. Vertex edges are drawn with two arrows allowing the discontinuity at the head and tail of the loop to be shown. The thick line shows the loop path along which the arrows has been flipped. The loop closes when the loop head (thick arrow) hits the loop tail (vertical bar).

vertices are joined, however for traditional vertex models nearest-neighbor vertices are joined together. The Monte Carlo algorithm discussed here always flips two (or zero) arrows on a vertex, thus it is limited to models where an even number of arrows are pointing away from each vertex. Most vertex models of interest obey this rule.

In visualizing the directed-loop Monte Carlo algorithm, originally developed for quantum systems [4], it is helpful to cut every edge into two pieces, each piece having an arrow belonging to a specific vertex, Fig. 1] For a valid vertex configuration the arrows on the two parts of an edge must have the same orientation. The directed-loop algorithm is as follows: Pick a random vertex $v_{1}$ and a random edge belonging to that vertex. Based on these choices select in a probabilistic manner another edge belonging to $v_{1}$ and name that the out-edge. Then flip the arrows on both the part of the in-edge and the part of the out-edge belonging to $v_{1}$. This introduces two discontinuities in the arrow configurations on the edges, one on 


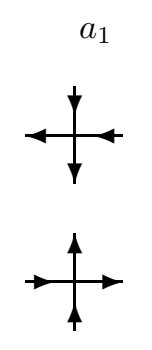

$a_{2}$

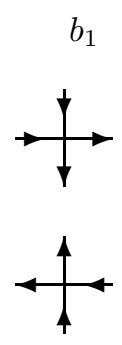

$b_{2}$

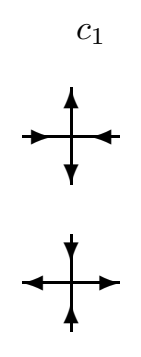

$c_{2}$
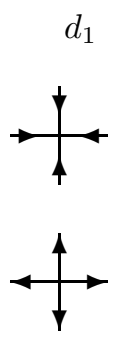

$d_{2}$

FIG. 2: The vertices of the eight-vertex model and their statistical weights.

the starting in-edge and another one on the out-edge. The new configuration is thus not an allowed vertex configuration. To repair this, the out-edge discontinuity is moved by repeating the procedure on the vertex connected to the out-edge $v_{2}$, this time using the out-edge of $v_{1}$ and the in-edge on $v_{2}$. The process is stopped when the out-edge selected is the starting edge, thus healing all discontinuities. In this way arrows are flipped as a loop is constructed, and a new allowed vertex configuration is arrived at when the loop closes.

In order to determine the probabilities for selecting out-edges and to see how detailed balance is satisfied one needs to consider also the probability for the reverse update. The reverse update consists of traversing the same loop in the opposite direction while flipping arrows back. As is explained in detail in Ref. [4], detailed balance is satisfied for the whole loop construction, if detailed balance is satisfied in each edge selecting step, for which the criterion is as follows: Let $w$ be the weight of the vertex $v$ before edge-flips, then the probability $P(v, i \rightarrow o)$ for exiting at the out-edge $o$, given that the in-edge is $i$, should satisfy

$$
w P(v, i \rightarrow o)=w^{\prime} P\left(v^{\prime}, o \rightarrow i\right),
$$

where $w^{\prime}$ is the weight of the vertex $v^{\prime}$ obtained by flipping the arrows on edges $i$ and $o$ belonging to the vertex $v$. Notice that $P\left(v^{\prime}, o \rightarrow i\right)$, on the right hand side, describes an edge-selecting step in the reverse update process where the loop is traversed in the opposite direction to that described on the left hand side. The loop construction should not terminate in the edge-selecting step, thus

$$
\sum_{o} P(v, i \rightarrow o)=1
$$

where the sum is taken over all possible out-edges, including the in-edge $i$.

This algorithm resembles closely the ice model algorithm invented by Rahman and Stillinger [5], generalized to arbitrary couplings by Barkema and Newman [6]. In fact, at the point in parameter space where all vertex weights are equal our algorithm is identical to the longloop version of the ice model algorithm. However away

from this point, Barkema and Newman's algorithm involves accepting or rejecting the loop after it has been constructed. The directed-loop algorithm has no such accept/reject step. A comparison of integrated autocorrelation times for the directed-loop algorithm and the short-loop algorithm of Barkema and Newman are shown in Fig. 3] The autocorrelation times are measured in units of lattice sweeps. One lattice sweep corresponds to a number of elementary loop moves such that on average each vertex on the lattice have been visited twice. In defining visited we include parts of the loop where the loop bounces off a vertex (relevant for the directed-loop algorithm) and the neck part of short-loops. Neither the bounces nor the short-loop-necks contribute to changes in the vertex configuration. However they are intrinsic parts of the algorithms and requires computer effort, and should therefore be accounted for.

The upper panel of Fig. 3 shows integrated autocorrelation times of the observable counting the number of $c$-type vertices in each configuration. This observables was chosen to compare with the performance results in Ref. [6]. While the integrated autocorrelation times are larger for the short-loop algorithm the scaling with system size appears to be equal for both algorithms. The lower panel shows integrated autocorrelation times for the total arrow-polarization in the y-direction. These scales much worse for the short-loop algorithm than for the directed-loop algorithm. This is to be expected from the fact that most loops accepted in the short-loop algorithm are small, while large loops that wind around the boundary of the lattice is needed to change the total polarization. These are not suppressed in the directed-loop algorithm, thus leading to better performance.

The Eqs. (1) and (2) form several coupled sets which in most cases are under-determined. There are thus many solutions for the out-edge selection probabilities $P$. Some general solutions and analysis of their efficiency for different quantum systems were reported in Ref. 7]. Here we employ the solution B in Ref. 7] to the eight-vertex model, but solutions for higher-vertex models are not hard to find as well. The allowed vertices for the eightvertex model and their statistical weights are shown in Fig. 2 To shorten notation, we consider the so-called symmetric case: the statistical weights, $a, b, c$, and $d$, of the allowed states are assumed to be invariant under the simultaneous reversal of all arrows. The generalization of the algorithm to the non-symmetric case can be performed easily.

Let $W_{1}, \ldots, W_{4}$ be the vertex weights $a, b, c, d$ of the eight-vertex model ordered so that $W_{1} \geq W_{2} \geq W_{3} \geq$ $W_{4}$. Then the probability for picking the out-edge on a vertex with weight $W_{i}$ resulting in a new vertex weight $W_{j}$ after flipping arrows is $t_{i j} / W_{i}$, where $t_{i j}=t_{j i}$ and the non-zero entries of the $4 \times 4$ matrix $t$ are

$$
\begin{aligned}
& t_{12}=\left(W_{1}+W_{2}-W_{3}-W_{4}\right) / 2, \\
& t_{13}=\left(W_{1}-W_{2}+W_{3}-W_{4}\right) / 2, \\
& t_{23}=\left(-W_{1}+W_{2}+W_{3}+W_{4}\right) / 2,
\end{aligned}
$$




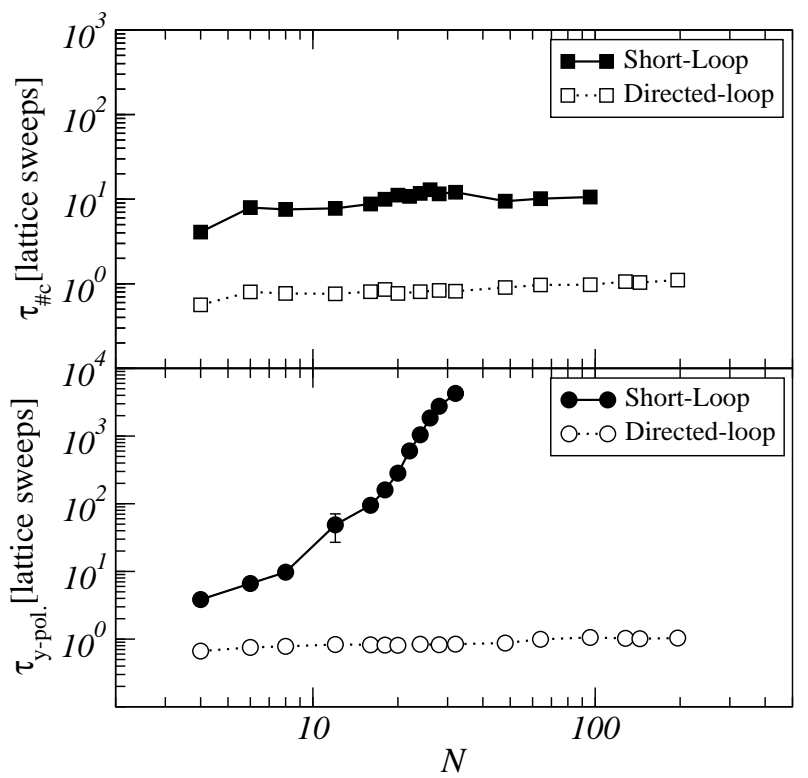

FIG. 3: Integrated autocorrelation times for number of $c$-type vertices (upper panel) and the total polarization in the $y$-direction (lower panel) for the directed-loop algorithm (open symbols) and the short-loop Barkema-Newman algorithm (closed symbols). The data shown is for the symmetric six-vertex model on an $N \times N$ square lattice with $\mathrm{PBC}$ and vertex weights $a=b=2$ and $c=1$.

$$
t_{14}=W_{4},
$$

when $W_{1}-W_{2}-W_{3}-W_{4} \leq 0$. Otherwise one needs to include bounces in which the out-edge coincides with the in-edge. In this case a solution can be chosen as follows:

$$
\begin{aligned}
t_{11} & =W_{1}-W_{2}-W_{3}-W_{4}, \\
t_{1 j} & =t_{j 1}=W_{j}, \quad j=2,3,4 \\
t_{i j} & =0, \quad \text { otherwise. }
\end{aligned}
$$

The directed-loop algorithm satisfies ergodicity as any configuration can be obtained from another configuration by flipping spins along a finite number of (possibly overlapping) loops. This process is exactly the directed-loop update, thus ergodicity follows.

The algorithm presented here has many similar features to the loop algorithm 8]. The loop algorithm breakup rules for the symmetric eight-vertex model can be chosen identical to Eq. (3), as can be seen from Ref. 9], whenever the weights are such that no bounces are needed in the directed-loop algorithm. However in parameter regimes where bounces are needed, the related feature in the loop algorithm is to "freeze" independent loops together. Bounces and "freezing" of loops are very different in how they act to change the configuration. While bounces is a local resistance to changing a vertex, "freezing" causes big non-local changes of the vertex configuration. There are also other differences: For general vertex models the set of non-freezing/bouncefree solutions is always smaller for the loop algorithm than for

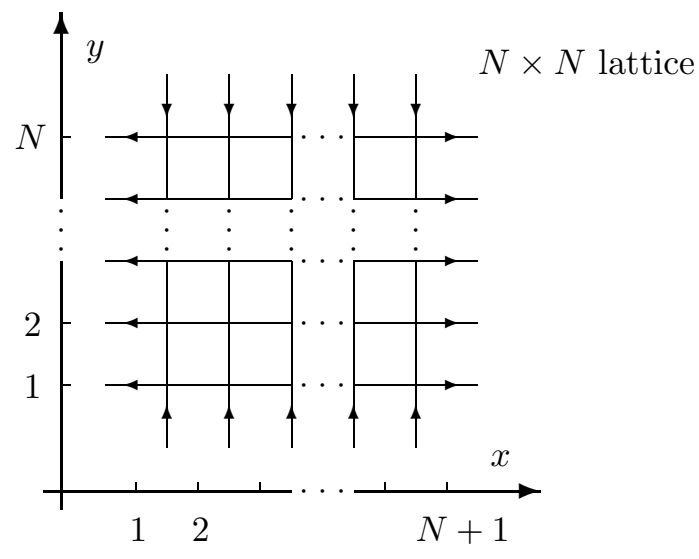

FIG. 4: The domain wall boundary conditions. Shown is an $N \times N$ lattice. The total number of vertices is $N^{2}$. The $x$ and $y$ coordinates take integer values at the midpoints of the horizontal edges.

the directed-loop algorithm. This allows the directedloop algorithm to be efficient in a larger region of parameter space than the loop algorithm. In particular this applies to the asymmetric eight-vertex model.

Note that the need for bounces is generally not so crucial for higher-vertex models with many weights of the same magnitude, thus we expect that the directed-loop algorithm should work well in simulating these. Note also that an algorithm based on the directed-loop idea was recently demonstrated to be effective in simulating classical integer-valued link-current models [10].

\section{SIX-VERTEX MODEL WITH THE DWBC}

The six-vertex model with the DWBC was introduced in Ref. 11] in connection with the calculation of the correlation functions for exactly solvable $1+1$ dimensional models [3]. Here we recall the definition of the model in brief, referring for further details to the Ref. 12] where a more detailed description of the model and a comprehensive list of references are given.

The model is defined on an $N \times N$ square lattice; the thermodynamic limit corresponds to $N \rightarrow \infty$. There are six possible states at each vertex: one should set $d=0$ in the eight-vertex model defined above, Fig. 2 The model is symmetric: the statistical weights, $a, b$, and $c$, of the allowed states are assumed to be invariant under the simultaneous reversal of all arrows. Hence, the model is characterized by only two parameters, which can be taken to be $a / c$ and $b / c$. We set $c=1$ henceforth.

The DWBC imply that all arrows on the top and bottom of the lattice are pointing inward, while all arrows on the left and right boundaries are pointing outward, Fig. 4

To investigate the spatially inhomogeneous behavior of this model we focus on the polarization, $\chi_{N}(x, y)$ 12, 13], 


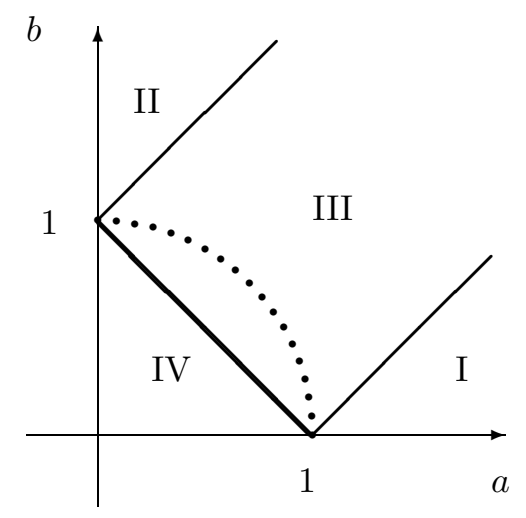

FIG. 5: The phase diagram of the six-vertex model in terms of the weights $a$ and $b$. One has $\Delta>1$ in the regions I and II, $-1<\Delta<1$ in the region III, and $\Delta<-1$ in the region IV. The dotted quartercircle corresponds to $\Delta=0$.

which is the ensemble average of the arrow direction on the edge with coordinates $(x, y)$ on the $N \times N$ lattice. The coordinate system used is shown in Fig. 4 Due to the symmetry of the model it is sufficient to consider the polarization of the horizontal arrows only. The value +1 $(-1)$ is assigned to an arrow pointing to the right (left) and the ensemble average is assumed to be normalized by dividing by the partition function. Therefore, $\chi_{N}$ lies between -1 and 1 .

Obviously, $\chi_{N}$ is independent of the coordinates of the edge in case of PBC. For these boundary conditions $\chi_{N}$ is known in the thermodynamic limit, and exhibits ferroelectric order, antiferroelectric (AF) order or no order, depending on the position on the $(a, b)$ plane. Thus, three phases exist in the six-vertex model with $\mathrm{PBC}$ : ferroelectric, antiferroelectric, and disordered phase. In Fig. $[5$ the phase diagram on the $(a, b)$ plane for the model with PBC is plotted (cf., Fig. 8.5 of Ref. 2]).

Introduce a parameter $\Delta$ by the formula

$$
\Delta=\frac{a^{2}+b^{2}-1}{2 a b} .
$$

The case $\Delta>1$ (regions I and II in Fig. 5) corresponds to the ferroelectric phase; the case $-1<\Delta<1$ (region III in Fig. 5) to the disordered phase; the case $\Delta<-1$ (region IV in Fig. 5) to the AF phase.

Fig. 5 may be considered as the phase diagram for the model with the DWBC, in the sense that the free energy takes a different analytic form in the regions I through IV (see Ref. 14] for details). But, in case of the DWBC the polarization $\chi_{N}$ depends on the position of the edge. In the next section we show numerical results for the polarization $\chi_{N}(x, y)$ of the horizontal arrows as the parameters $a$ and $b$ are varied.

Making use of the directed-loop algorithm described in the previous section for simulation of the model with the DWBC one should treat vertices belonging to the boundary and the bulk vertices differently. In the bulk one finds by setting $d=W_{4}=0$ and $c=1$ in Eqs. (3), that bounces are only necessary when $a+b<1$ or $|a-b|>$ 1. For the boundary vertices the loop is not allowed to exit on the boundary edges, because the arrows on these edges are fixed by the boundary conditions. This leads to more restricted equation sets (many $W$ 's are equal to zero) for the boundary vertices and generally requires the inclusion of bounce processes.

Another important point should be mentioned is that the DWBC do not violate the ergodicity of the algorithm even though loops which wind around the boundaries are excluded. These winding loops are needed in order to change the net polarization in the $x$ - or $y$-direction. However, one can verify that the boundary conditions restricts the net polarization in both these direction to be zero for any configuration, so winding loops are not necessary to sample the full configuration space allowed by the boundary conditions.

\section{RESULTS}

In this section we present the results of the simulations for the polarization $\chi_{N}(x, y)$ in the disordered, antiferroelectric and ferroelectric phases.

(i) Disordered phase: $-1<\Delta<1$. First consider the particular case $\Delta=0$ (dotted quartercircle in Fig. 15). An exact expression for $\chi_{N}(x, y)$ in this case was obtained by Kapitonov and Pronko 15] recently. To check our algorithm we have compared results for the polarization at the point $a=b=1 / \sqrt{2}$ with the exact results of Ref. [15]. The comparison can be seen in Fig. 6] where the polarization is shown as a function of $x$ for different values of $y$ and system sizes, $N$. One can clearly see that the boundary values of the polarization, \pm 1 , extends a finite distance into the bulk and this distance depends on $y$. The areas where the polarization stays at its boundary values are termed "frozen" regions. Going further into the bulk, there is a transition to a "disordered" region, where apart from small wiggles due to the finite system size, the polarization changes smoothly. It is interesting to note that there never is any extended regime where the polarization is zero, as is the case for PBC. The transition between the "frozen" and "disordered" regions gets sharper as the system size is increased, as can be seen by comparing the two panels in Fig. [6]

It is convenient to visualize the behavior of the polarization using greyscale plots, where greyvalues are assigned to values of $\chi_{N}(x, y)$ and each point $(x, y)$ corresponds to a location of the midpoint of a horizontal edge following the layout described in Fig. 4 For $a=b=1 / \sqrt{2}$ such a plot is shown in Fig. 7/a). The four "frozen" corners are clearly apparent. In these regions, the vertices are all of the same type, and are, from upper left to bottom right, $a_{1}, b_{1}, b_{2}, a_{2}$, respectively. To measure the area of the "frozen" regions, we define a threshold value $\epsilon=0.08$, such that points $(x, y)$ where $\left|\chi_{N}(x, y)\right|>1-\epsilon$ are judged to be in a "frozen" region. 


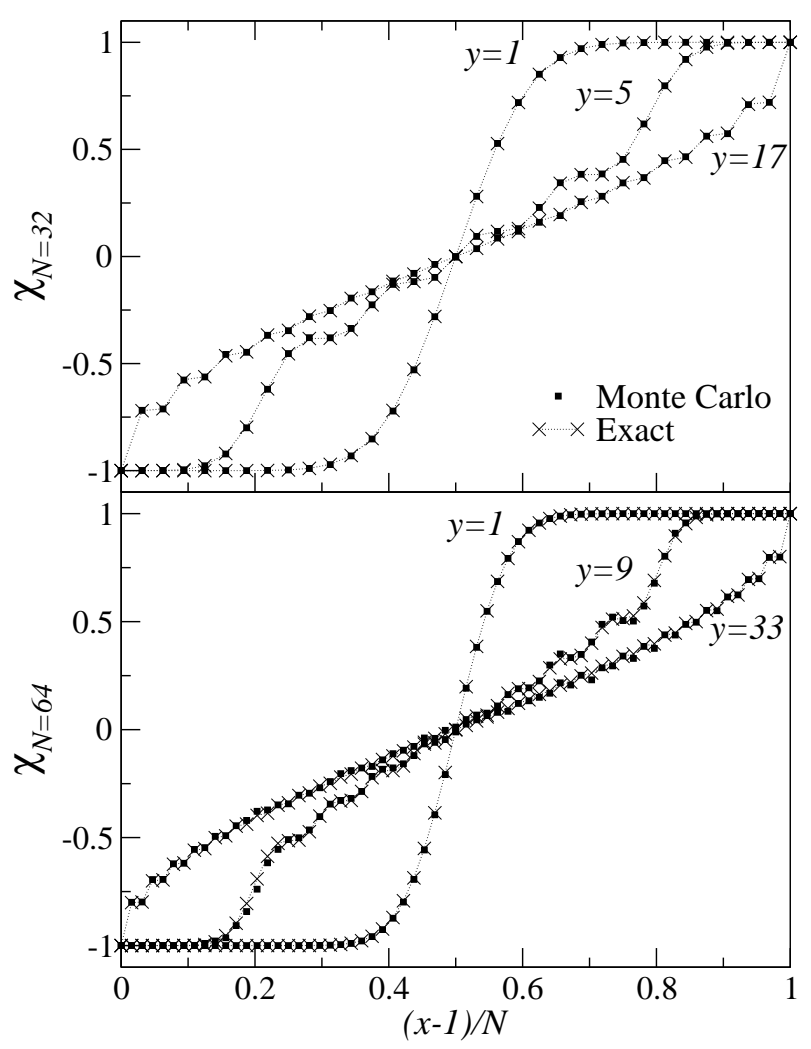

FIG. 6: Polarization $\chi_{N}(x, y)$ as a function of $x$ for different values of $y$. Vertex weights $a=b=1 / \sqrt{2}$. Results for two different system sizes are shown: $N=32$ (upper panel) and $N=64$ (lower panel). The filled symbols are Monte Carlo results, while the crosses are exact results gotten from Ref. [15]. The dotted lines are guides to the eye.

Applying this we find that each "frozen" corner is $4.6 \%$ of the total area. This value changes relatively little changing the value of $\epsilon$.

Going away from the $\Delta=0$ curve, let us follow along the diagonal, $a=b$, towards $\Delta=\infty$ first, Fig. 7 As the values of the vertex weights $a$ and $b$ increase, the area of the "frozen" regions decreases. We find that with $\epsilon=0.08$ each frozen corner in $(b)$ is $4.0 \%$ of the total area, and $2.8 \%$ in (c). For very large values of $a=b$, the polarization $\chi_{N}(x, y)$ increases linearly from -1 to 1 as $(x-1) / N$ goes from 0 to 1 , independent of $y$, as can be seen in Fig. 17(d). This is consistent with what is expected from an ensemble of configurations with the smallest possible number of $c$-type vertices: $N$ ! configurations each with a single $c$-type vertex on every row and column.

Consider now $a \neq b$. Because of the symmetry of the phase diagram, Fig. [5 one can choose $b>a$ without loss of generality. The weights of the vertices in the four "frozen" corners are no longer equal, and the "disordered" region distorts into an oblong shape oriented along the diagonal with large corners of $b_{2}$ and $b_{1}$ vertices, see Fig. 8 The simulations for $a=1 / 4$ and $b=\sqrt{15 / 16}$ are shown in Fig. 8 (a). The width of the oblong region

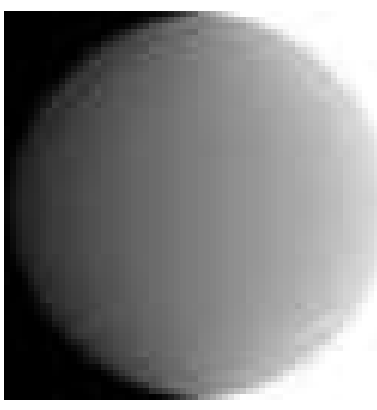

(a)

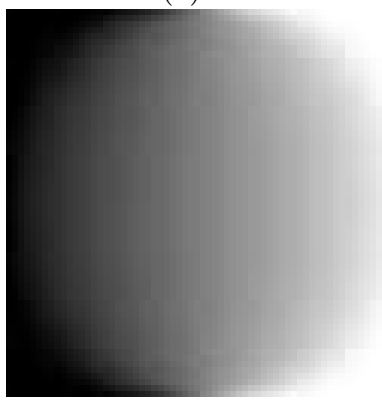

(c)

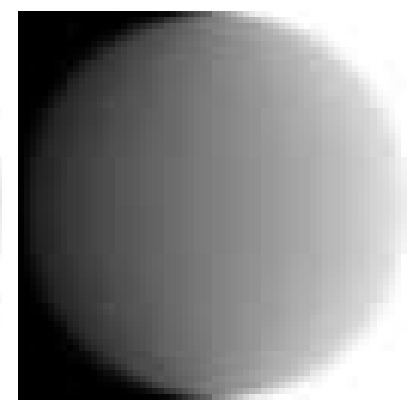

(b)

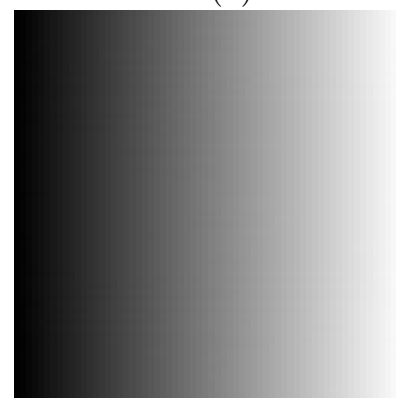

(d)

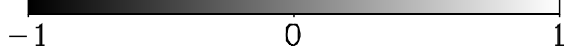

FIG. 7: Greyscale plot of the polarization $\chi_{N}(x, y)$ for $N=64$ in the disordered phase. Vertex weights are equal, $a=b$, and run through the values $1 / \sqrt{2}, 1,3,100$ for figures (a)-(d), respectively. The corresponding values of $\Delta$ are $0,1 / 2,17 / 18$, $1-5 \cdot 10^{-5}$.

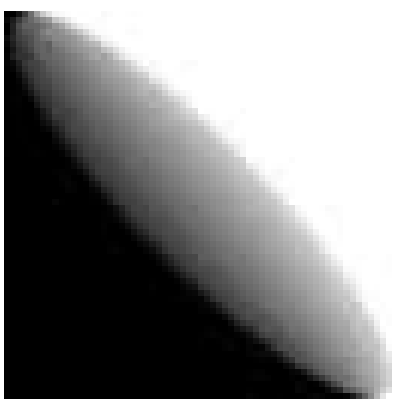

(a)

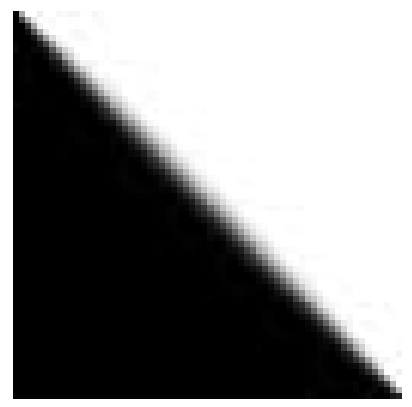

(b)

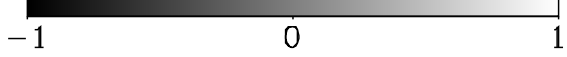

FIG. 8: Greyscale plot of the polarization $\chi_{N}(x, y)$ for $N=$ 64 . The weight $a=1 / 4$, while the weight $b$ is chosen to be $b=$ $\sqrt{15 / 16}(\Delta=0$, disordered phase) in figure (a) and $b=5 / 4$ $(\Delta=1$, the boundary between disordered and ferroelectric phases) in figure (b).

shrinks as $b$ increases keeping $a$ fixed, $a=1 / 4$, and becomes very thin at the boundary to the ferroelectric region, as can be seen in Fig. 8 (b). Along this boundary, $b=a+1$, the width of the oblong region expands as $a$ increases with $N$ being constant.

(ii) Antiferroelectric phase: $\Delta<-1$. The simulations 


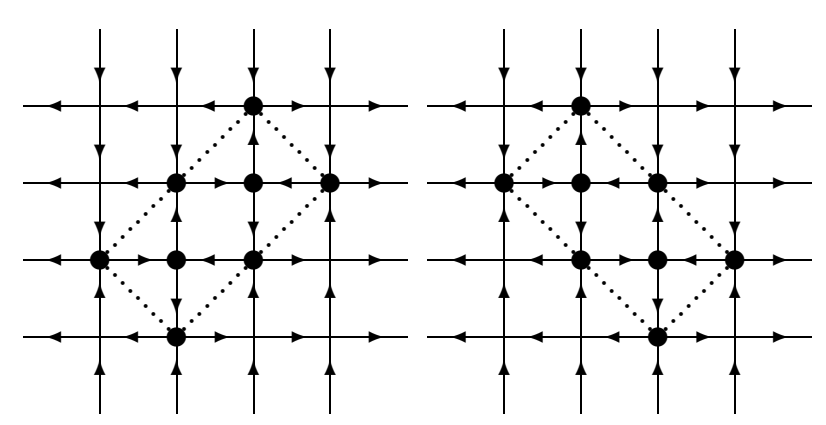

FIG. 9: The two configurations having maximum number of the $c$-type vertices. These vertices are marked by filled circles. The size of the lattice is $4 \times 4$.

in the AF phase are less efficient than in the disordered phase. This is partly due to the presence of the bounce processes also for bulk vertices, but another feature which makes the simulations difficult in this phase is the degeneracy of the two types of AF orders. In the AF phase it becomes energetically favorable to have a maximum possible amount of $c$-type vertices, which is achieved by placing $c$-type vertices in a diamond placed in the center of the lattice. For an even $N$ this diamond can be placed in two equivalent places differing only by one lattice spacing, as shown in Fig. 9 The Monte Carlo algorithm is however slow in tunnelling between these configurations, and this sets a limit to its performance. For odd $N$ there is no such a degeneracy and the simulations are more efficient. Greyscale plots of the polarization for $a=b=1 / 2$ and $a=b=3 / 8$ are shown in Fig. 10. We have plotted results for both even and odd $N$.

One can see that the "disordered" region have a diamond-like shape, which is consistent with the domination of the $c$-type vertices in the AF phase. As $a=b$ decreases $(\Delta \rightarrow-\infty)$, the shape of the "disordered" region should converge to the one shown in Fig. 9 that is, the boundaries of the "disordered" region should become more and more straight. But, this convergence appears to be rather slow and it is not easy to see it from Fig. 10. What one can clearly see from Fig. 10 is the difference between odd and even $N$. For odd $N$ AF oscillations are clearly visible in the center of Figs. 10/(c) and (d), while they are much weaker for even $N$, Figs. 10(a) and (b), reflecting the degeneracy mentioned above. These difference between even and odd $N$ can also be clearly seen from Fig. 14 For odd $N$ AF oscillations are weaker at $a=b=1 / 2$ than at $a=b=3 / 8$.

For $a \neq b$ greyscale plots are shown in Fig. 111 Here $\mathrm{AF}$ oscillations in the middle of the plot are visible for $a=1 / 4$ and $b=1 / 2$, Fig. 111(a), while they have almost vanished at the boundary between the $\mathrm{AF}$ and disordered phases, Fig. 11(b).

(iii) Ferroelectric phase: $\Delta>1$. The behavior of the polarization in this phase is essentially the same as shown in Fig. 8 Vertices of type $b$ dominate completely in the region II of the phase plane Fig. 5 while in the region

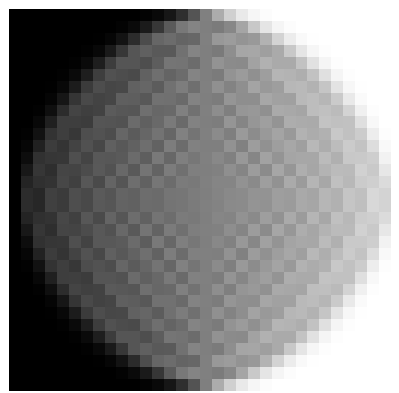

(a)

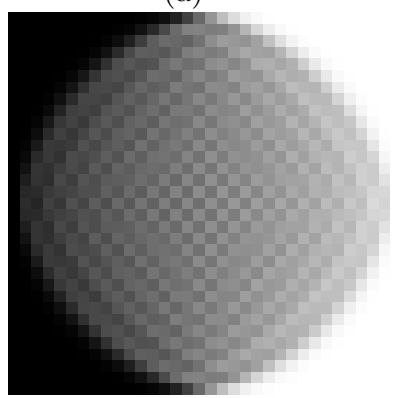

(c)

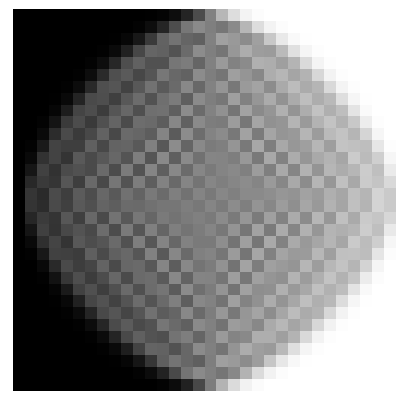

(b)

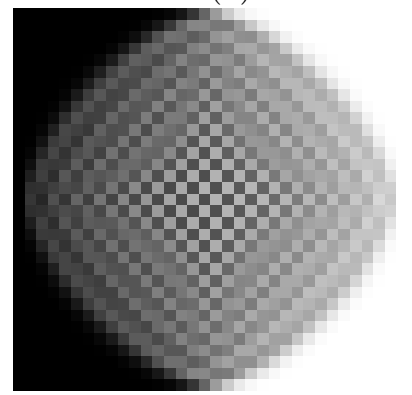

(d)

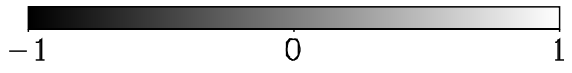

FIG. 10: Greyscale plot of the polarization $\chi_{N}(x, y)$ for two different system sizes: $N=32$ in figures (a) and (b) and $N=33$ in figures (c) and (d). Vertex weights are equal, $a=b$, and take the value $1 / 2(\Delta=-1$, the boundary between disordered and AF phases) for figures (a) and (c), and the value $3 / 8(\Delta=-23 / 9$, AF phase) for figures (b) and (d).

I of the phase plane the dominant vertices are those of type $a$. If one goes along the phase boundary, $b=a+1$, towards $a=\infty$, the widths of the "disordered" region is increased, as we have mentioned in the end of the part (i) of this Section.

The exact expression is known [12] for the polarization along the boundary, $\chi_{N}(x, 1)$. Comparing our Monte Carlo data to this expression we find that in no cases is the absolute difference bigger than 0.016 , which is comparable to the statistical errors of our simulations.

\section{DISCUSSION}

We have considered the phase diagram of the model for the given $N$. Now, discuss the following problem: what happens with $\chi_{N}(x, y)$ in the thermodynamic limit, $N \rightarrow \infty$ ? It is natural to expect that differences in the behavior of the polarization in the different parts of the phase plane, Fig [5] become more pronounced as $N \rightarrow \infty$. As one can see in Fig. [6] the wiggles in the "disordered" region decrease with $N$ increasing, and this is, indeed, the case for all the points $(a, b)$ lying in the disordered phase $(-1<\Delta<1$, region III of the phase plane, Fig. 5) and checked in our simulations. We expect that these wiggles, 


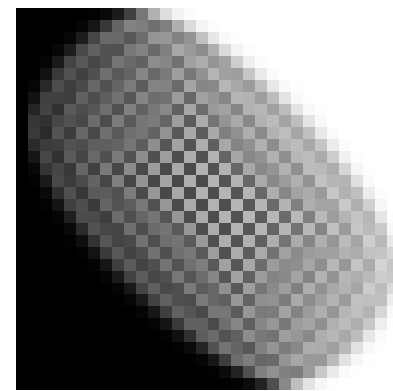

(a)

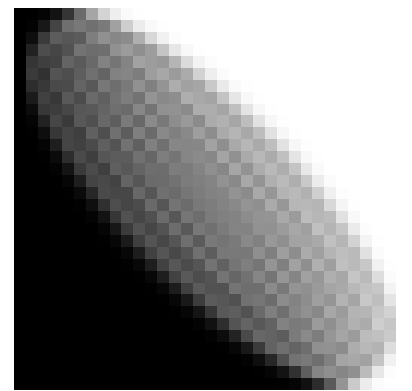

(b)

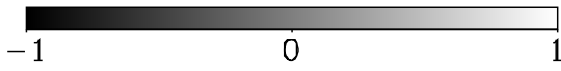

FIG. 11: Greyscale plot of the polarization $\chi_{N}(x, y)$ for $N=$ 32. The weight $a=1 / 4$ while the weight $b$ is chosen to be $b=1 / 2(\Delta=-11 / 4$, AF phase $)$ in figure (a) and $b=3 / 4$ ( $\Delta=-1$, the boundary between disordered and AF phases) in figure (b).

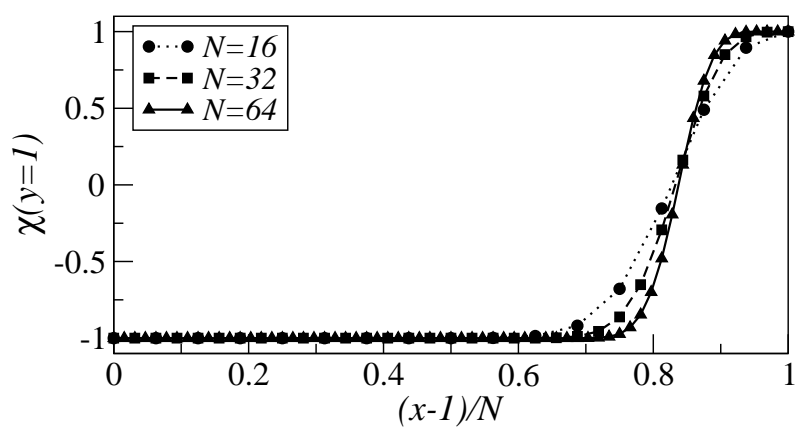

FIG. 12: Boundary polarization $\chi_{N}(x, 1)$ is shown for three system sizes, $N=16,32$, and 64 . Vertex weights $a=1 / 4$ and $b=3 / 4(\Delta=-1$, the boundary between disordered and AF phases). Note the steepening of the curve as $N$ increases.

coming from the antiferroelectrically ordered configurations, should vanish completely in the thermodynamic limit for this phase. The next conjecture we want to make is on the behavior of the polarization along the boundary, $\chi_{N}(x, 1)$. It is known that for $\Delta=0$, as well as at the point $a=b=1$, the boundary polarization becomes the Heaviside step function in the thermodynamic limit [12, 16]. We conjecture that this is the case for the whole disordered phase; the position of the discontinuity will depend on the ratio between $a$ and $b$. We present Fig. 12 to support this conjecture.

Furthermore, note that for $a=b=1 / \sqrt{2}$ there is a mapping (see, e.g., Ref. [14]) of the six-vertex model with the DWBC onto the so-called model of domino tilings of the Aztec diamond. The thermodynamic behavior of the latter model was investigated in Refs. [17]. It shows the same features as in Fig. I7 a): the tilings are ordered (frozen) in the corners of the diamond, while going inside one falls into the "disordered" region. All these features were named the "Arctic Circle Theorem", since the shape

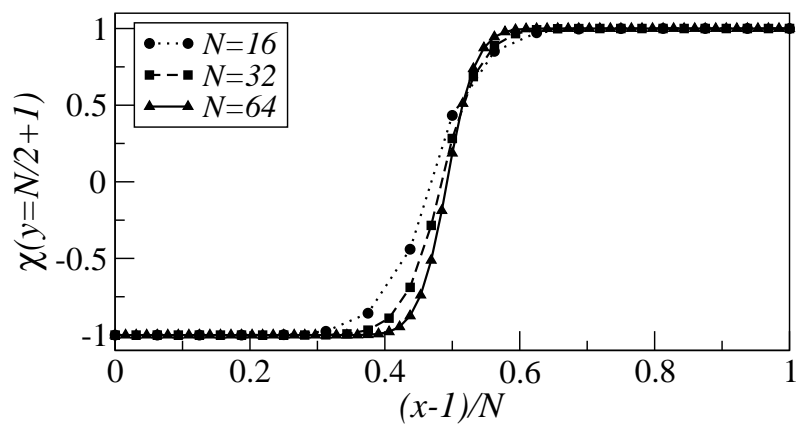

FIG. 13: Polarization $\chi_{N}(x, y=N / 2+1)$ is shown for three system sizes, $N=16,32$, and 64 . Vertex weights $a=1 / 4$ and $b=5 / 4(\Delta=1$, the boundary between disordered and ferroelectric phases). Note the steepening of the curve as $N$ increases.

of the boundary between the "frozen" and "disordered" regions is circular. The transition between "frozen" and "disordered" regions is step-like, with the height of the step function depending on the coordinates $x$ and $y$.

We expect the analogue of the Arctic Circle Theorem to take place for the whole disordered phase, $-1<\Delta<$ 1: there should be the "frozen" regions, "disordered" region, and a sharp transition between them. We expect also that the profile of the boundary between the "frozen" and "disordered" regions is circular for $a=b$, even though there is no obvious symmetry protecting this statement. Note that the very "smeared" profile in Fig. 7(d) does not contradict our hypotheses because $N=64$ is relatively small compared to the values of the vertex weights $a$ and $b$, and is thus far from the thermodynamic limit for this point of the phase diagram.

For the ferroelectric phase, $\Delta>1$, the greyscale plot Fig. 8 (b) together with the scans shown in Fig. 13 leads to the natural conjecture: in the whole region II of the phase plane, Fig. [5] a sharp discontinuity from a "frozen" domain with $b_{1}$ vertices to the one with $b_{2}$-vertices takes place in the thermodynamical limit. In the region I the behavior is essentially the same, one should simply use $a$-type vertices instead of the $b$-type.

To this end, consider the antiferroelectric phase, $\Delta<$ -1 . We expect the step-like behavior of the boundary polarization, $\chi_{N}(x, 1)$ in this phase in the thermodynamic limit, as well as the existence of the "frozen" regions in the corners. Our statements on the behavior of the polarization deep inside the lattice are more speculative. For $a=b$ and even $N$ the height of the AF oscillations decreases, while for odd $N$ these oscillations seem to be non-vanishing in the large $N$ limit, see Fig. 14 Our belief is that there is a finite region with AF order for odd $N$, as $N \rightarrow \infty$, while for even $N$ the polarization exhibits no such an order.

Finally, we would like to stress that the directed-loop algorithm can also be applied to study the six-vertex model with any boundary conditions, and the higher- 


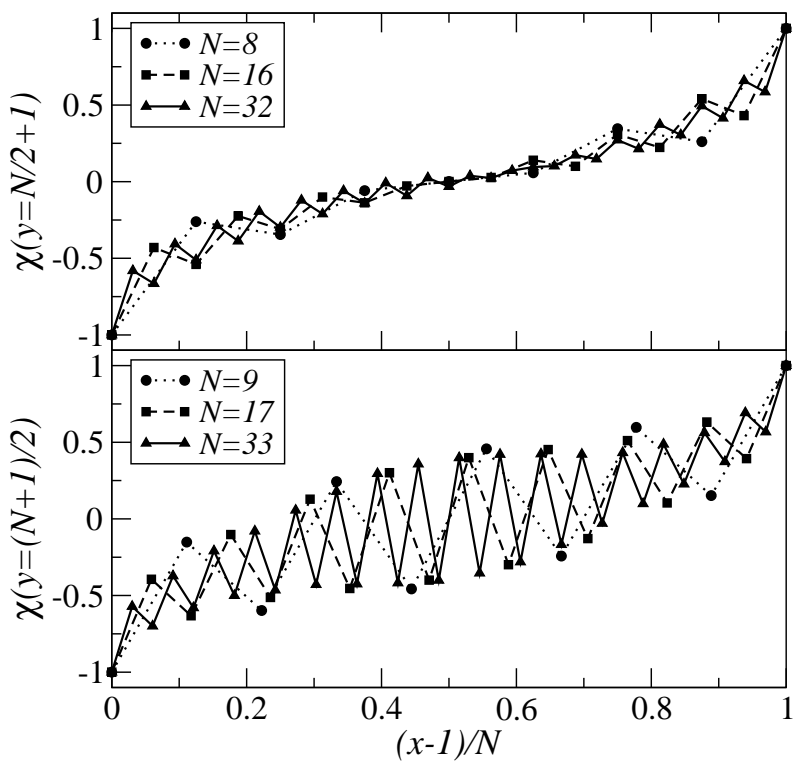

FIG. 14: Polarization $\chi_{N}(x, y)$ along lines of constant $y$, where $y=N / 2+1, y=(N+1) / 2)$ for even and odd $N$ respectively, is shown for $N=8,16$, and 32 (upper panel) and $N=9,17$, and 33 (lower panel). Vertex weights $a=b=3 / 8$ $(\Delta=-29 / 9$, AF phase $)$. vertex models. These could help in solving the problems for which the analytical methods are difficult to apply. For example, the six-vertex model with any boundary conditions can be considered as a model for a description of interface roughening of a crystal surface [18]. An important point in studies Refs. 18] is the existence of exact analytical results for the six-vertex model with PBC 1, 2]. Therefore, numerical data referring to other boundary conditions than PBC could give a new insight for these studies.

\section{Acknowledgments}

We thank V.V. Cheianov and A.G. Pronko for useful discussions and the authors of the work [15] for providing us with their data. M.B. Zvonarev's work was supported by the Danish Technical Research Council via the Framework Programme on Superconductivity. Monte Carlo calculations were in part carried out using NorduGrid, a Nordic facility for Wide Area Computing and Data Handling.
[1] E.H. Lieb, Phys. Rev. 162, 162 (1967); Phys. Rev. Lett. 18, 1046 (1967); 19, 108 (1967); B. Sutherland, ibid. 19, 103 (1967).

[2] R.J. Baxter, Exactly Solved Models in Statistical Mechanics (Academic Press, San Diego, 1982).

[3] V.E. Korepin, N.M. Bogoliubov, and A.G. Izergin, Quantum Inverse Scattering Method and Correlation Functions (Cambridge University Press, Cambridge, 1993).

[4] O.F. Syljuåsen and A.W. Sandvik, Phys. Rev. E, 66, 046701 (2002).

[5] A. Rahman and F.H. Stillinger, J. Chem. Phys. 57, 4009 (1972).

[6] G.T. Barkema and M.E.J. Newman, Phys. Rev. E 57, 1155 (1998).

[7] O.F. Syljuåsen, Phys. Rev. E, 67, 046701 (2003).

[8] H.G. Evertz, G. Lana and M. Marcu, Phys. Rev. Lett. 70, 875 (1993).

[9] N. Kawashima, J. Stat. Phys. 82, 131 (1996).
[10] F.Alet and E.S. Sørensen, Phys. Rev. E, 68, 026702 (2003).

[11] V.E. Korepin, Commun. Math. Phys. 86, 391 (1982).

[12] N.M. Bogoliubov, A.G. Pronko, and M.B. Zvonarev, J. Phys. A 35, 5525 (2002).

[13] N.M. Bogoliubov, A.V. Kitaev, and M.B. Zvonarev, Phys. Rev. E, 65, 026126 (2002).

[14] P. Zinn-Justin, Phys. Rev. E 62, 3411 (2000).

[15] V.S. Kapitonov and A.G. Pronko, private communication.

[16] D. Zeilberger, NY J. Math. 2, 59 (1996).

[17] W. Jockusch, J. Propp, and P. Shor, e-print math.CO/9801068 H. Cohn, N. Elkies, and J. Propp, Duke. Math. J. 85, 117 (1996).

[18] H. van Beijeren, Phys. Rev. Lett. 38, 993 (1977); E. Carlon, G. Mazzeo, and H. van Beijeren, Phys. Rev. B 55, 757 (1997). 